\title{
Fibrinolytic therapy of coronary thrombosis
}

\author{
H. A. DEWAR \\ From the Royal Victoria Infirmary, Newcastle-on-Tyne
}

The idea of using fibrinolytic agents in the treatment of coronary thrombosis is attractive and obvious. The onset of the disease is acute and clearly defined, the disease itself is a very serious one, present methods of treatment leave so much to be desired, and even if one were unable to save cardiac muscle from necrosis, the lesser goal of restoring to the patient a patent coronary artery is well worth pursuing. For all these reasons, when in 1959 Ruegsegger, Nydick, Hutter, Freiman, Bang, Cliffton, and LaDue, provided the experimental basis for success and Messrs. Merck, Sharp, and Dohme provided an apparently safe and active fibrinolytic preparation, we seized the opportunity of embarking on a controlled trial. Their experimental basis was that in dogs segments of coronary artery were isolated between ligatures and the sequestrated blood in them was then clotted with thromboplastin. At various times after the ligatures were removed the animals were perfused with the fibrinolytic agent. By coronary angiography the clots were clearly shown to dissolve in a high proportion of the animals treated. If more than a few hours had elapsed between clotting and lysis the heart muscle was not saved from death but the area of infarction was smaller than in the controls. The surrounding inflammatory reaction was less, and what Ruegsegger et al., called 'microthrombi', i.e., occlusion of the small blood vessels, around the infarct were not found. The fibrinolytic preparation they used was a human plasma whose plasminogen had been activated by streptokinase and its activity was said to depend solely on its preformed plasmin content and not upon its residue of activator which, indeed the manufacturers claimed, was not any longer present. This same preparation, Thrombolysin, is what we used for our clinical trial. It was extremely expensive, but was remarkably free of unpleasant side-effects. Fever began a bit sooner in the treated group than in the control, but that was all. Temperatures were not higher, there were no rigors, and the patients did not suffer.

Details of our trial have already been published, (Dewar, Stephenson, Horler, Cassells-Smith, and Ellis, 1963) but briefly we started a pilot trial taking alternate patients on admission but, once we foundw the substance was safe and active, we became a little more respectable and completed the trial by the better random allocation method, using prepared $\vec{v}$ envelopes containing the word 'treated' or 'control' $\omega$ The drug was given by intravenous drip in large bute् arbitrary dosage for an also arbitrary period of 12 hours. It was followed by anticoagulant therapy with heparin and phenindione. The total of patients numbered 75, 38 treated and 37 controls. Criteria of success were the mortality, inevitably small (17 cases), but necropsies were obtained in nearly all of them. Other criteria were the clinical progress of the patient,,+ analysis of the electrocardiograms, and a mostr elaborate analysis of the curves of release of transaminase into the blood stream. I think we are possibly the only workers who have records of $57 \%$ such curves taken four-hourly day and night for $\frac{\mathrm{D}}{2}$ three days and then daily for seven more.

And what were our results? Briefly, whollyo negative. Of the treated group, nine patients died, of the untreated eight. From the 14 necropsies the hearts were all examined by the one pathclogist who was not told beforehand from which group they came. 0 He did not find fewer thrombi in the main arteries; he did not find fewer thrombi in the small vessels though he did find the capillaries more dilated in theo treated series. This is the opposite to what he should $₹$ have found. Nor did he find less inflammation? around the infarct. In our analyses of the electro- $D$ cardiograms we found slightly less ST elevation (a을. mere $\frac{1}{2} \mathrm{~mm}$.) at 24 hours in the treated group but non difference otherwise. In our analyses of the trans- - aminase curves we found no significant differences $N$ whatever.

Now the interesting question to ask is, 'Why did weo fail?' I have suggested possible reasons as follows 0 (1) Conditions were unfavourable for thrombolysis, namely, (a) thrombolytic agent too weak, given in $\stackrel{+}{+}$ inadequate dosage, or ineffective at the required 0 site; (b) thrombi not lysable owing either to theirö innate nature or to the late start of treatment. $\stackrel{\odot}{\circ}$ (2) Thrombi lysed but evidence of lysis was not ob-市 tained because $(a)$ too few patients were included in the trial, (b) coronary arteriography was not done, 
and $(c)$ muscle damage was already too advanced. (3) Thrombi are not important anyway.

The thrombolytic agent may have been too weak: preformed plasmin is not the ideal substance, for there are neutralizing anti-substances in the blood stream and if the dosage is large enough to overcome these (as we were able to show our dosage did in $86 \%$ of those tested) it may well then simply exert its proteolytic action on the plasma fibrinogen and leave the occlusion of the coronary artery relatively untouched. Activator may be better, for fibrin clots absorb plasminogen and so contain the seeds of their own disintegration. It is a pity that activator hitherto has so often been toxic because patients with coronary thrombosis will not tolerate rigors. It is also possible that it was ineffective when given intravenously, and Boucek and Murphy (1960), who injected their fibrinolytic agent at the root of the aorta, may be justified in claiming much better results. I have not plucked up courage to do this, and the fact that they have not published a larger series than their initial small one makes one wonder if their further experiences have been disappointing.

Whether treatment was started too late for the thrombi to be lysed is hard to say. The average lapse of 6.2 hours between the onset of the myocardial infarct and the commencement of treatment is probably as short as can be found in a general hospital in Great Britain, so that if further progress is to be made along those lines the thrombolytic agent would have to be put in the hands of the general practitioner or even of the patient.

Whether the thrombi are really lysable is also hard to say; there is no doubt that the natural basis of coronary thrombosis differs in important respects from the experimental clots which Ruegsegger et al. (1959) introduced into the coronary arteries of their dogs and the natural platelet thrombi seen in man may well be more resistant.

So far as the number of patients in the trial were concerned, our statistical advisers assured us that this was not so and any worthwhile therapy would have shown itself already, and I think that this is probably true. Coronary arteriography was not done because of its innate danger, and if it is the only criterion by which one can judge whether one has done a patient any good it is doubtful if that good is worth doing. This equally applies to the muscle damage, for if this is already so advanced that no improvement by clearing the artery can be demonstrated, then this too makes one wonder if the treatment is worthwhile, though it must beadmitted that a patient with a patent coronary artery is better than one with a blocked artery no matter how much damage has been done.

Finally, I have put down that the thrombi are not intrinsically important, because this is the view of some pathologists. It is not my own view nor that of Professor Duguid who examined some of the patients for me, but I think that one must recognize that in most instances the actual thrombosis is the last act in a process of progressive atheromatous narrowing, so that even if this final occlusion is cleared the patient still has a vessel too narrow for the health of his muscle. It may well be that if fibrinolysis has a place in the treatment of coronary artery disease it may lie more in creating a state of continuous enhanced fibrinolytic activity in the blood stream of these patients than in applying an artificial agent at the moment when they have a thrombosis.

\section{REFERENCES}

Boucek, R. J., and Murphy, W. P. Jr. (1960). Amer. J. Cardiol., 6, 525. Dewar, H. A., Stephenson, P., Horler, A. R., Cassells-Smith, A. J., and Ellis, P. A. (1963). Brit. med. J., 1, 915.

Ruegsegger, P., Nydick, I., Hutter, R. C., Freiman, A. H., Bang, N. U., Cliffton, E. E., and LaDue, J. S. (1959). Circulation, 19, 7.

PROFESSOR DAVIDSON complimented Dr. Dewar and said that his paper was an example of how negative results should be given.

DR. FEARNLEY asked Dr. Dewar if he would be prepared to repeat his study with other reagents.

DR. STEWART wondered whether the dosage used by Dr. Dewar in his trial was optimal.

DR. DEWAR replied that if he were repeating the study he would undoubtedly estimate dosage requirements, although presenting considerable technical difficulty, but he felt that this was not the reason for the failure of his trial.

DR. MCNICOL pointed out that if fibrinolytic therapy were of real value in myocardial infarction this would have emerged in Dr. Dewar's trial despite the various problems which beset it. 